\title{
A New method for Image Segmentation in Heart Vessels Ultrasound Using Wavelet Transform
}

\author{
Ayyoob Jafari
}

\begin{abstract}
Automatic image segmentation and boundary detection of blood vessels is desirable for analysis and diagnosis of some disease. In this paper, we introduce a new image segmentation approach to boundary determination of blood vessels in ultrasound images. This new technique is a combination of a region based segmentation using Mumfordshah model and Gabor wavelet filter. This approach needs no initial approximation. Gabor wavelet filter is used to outcome complicated image intensity distribution in ultrasound images. The proposed method is used to determine boundaries in vessels ultrasound images. This method requires no training stage and relatively is fast.
\end{abstract}

Index Terms-Component, image segmentation, gabor filter, vessels ultrasound images

\section{INTRODUCTION}

Heart and vessel diseases are one of the main causes of that kills people in industrial societies. One of the main important failures is the stenosis of Coronary vessels of the heart. Because of its slow development, it is a quiet disease and it might only show itself when the persons has a sudden death or heart attack. The main cause of this disease is due to accumulation of Plaques in Coronary vessels which disturbs blood flow. Therefore, diagnosis methods for tightness of Coronary vessels and then taking some further steps into preventing from complete stenosis of blood arteries is very important.

Conventional methods used for diagnosis of blockage in heart include X-ray angiography which is known to be a high risk method that raises many problems and faults. Apart from it being an invasive approach, this method has no ability to detect stiffness of vessel and the amount of plaques on the vessel layer [1]. One of the most desirable approaches for analysis of vessels stenosis is the usage of ultrasound images which produces vessels images noninvasively. Ultrasound waves have no danger for human tissues and don't have the dangers caused by X-ray.

Extraction of information from blood vessels such as diameter, branch angel, length and movement profile in a cardiovascular cycle also be useful to asses the severity of the disease. So, blood vessel segmentation is an important stage in this process. The manual segmentation requires trained specialists, so appropriate automatic segmentation is always desirable. Blood vessel segmentation has received much attention in recent researches and different approaches such as vessel tracking [2], deformable model [3] and supervised classification [4], [5] are developed. In this paper

Manuscript received October 12, 2012; revised November 23, 2012

Ayyoob Jafari is with Biomedical Engineering Department, Islamic Azad University, Qazvin Branch, Qazvin, Iran (e-mail: Ajafari20@qiau.ac.ir) we used from a region based model which is called Mumford-shah (MS) model [6] in blood vessel segmentation. A modified MS model introduced by Xiaojun [7] is used in this research which has the ability to adopt to the complicated image intensity distributions which is usual in ultrasound images. Also, Gabor wavelet filter [8] is used to improve the results of proposed method. This approach is fast and there is no need to any type of training stage.

The organization of paper is as follows. In section II, we first describe the MS model basis and MS model used in this research. Section III describes the Gabor wavelet filter. Section IV shows our blood vessel segmentation algorithm. Experimental results are described in section $\mathrm{V}$ and we conclude paper in section VI.

\section{THE MUMFORD-SHAH APPROACH}

Mumford and Shah [6] proposed an image segmentation algorithm based on minimization of an energy function. This energy function is

$$
E(u, C)=\int_{\Omega}\left|u-u_{0}\right|^{2} d x d y+\mu \int_{\Omega \mid C}|\nabla u|^{2} d x d y+v \cdot|C|
$$

where $C$ is segmentation curve; $|C|$ is the curve length; $u_{0}$ is original image and $u$ is smooth approximation of original image. $\Omega$ is image domain and $\Omega \backslash C$ is image domain excluding the segmentation curve. If the MS energy function in eq.1 minimized, the image will be segmented into regions in order to boundary of each region is as short as possible and $u$ be a smooth approximation of $u_{0}$.

Using eq.1, segmentation problem is reduced to the problem of finding a piecewise smooth approximation of $u$. The functional $E(u, C)$ has been studied extensively and a large body of results has been obtained [9]-[11]. Some alternative solutions for this problem are suggested in [12]. Chan and Vesse used level set method for piecewise smooth approximation [13]. In their method, approximation of image density is done by smooth functions and MS model function is solved by three Partial Differential Equations (PDEs). PDE based proposed solution is a very time consuming approach. To outcome this problem, Chan and Vesse proposed the piecewise constant smooth approximation [14]. In new method, the image intensity inside object regions is approximated by constants and then there is no need to solve two PDEs. The image intensity inside regions is presented by the average intensity of the object and only one PDE should be solved for segmentation curve. In addition to constant approximation algorithm, other methods such as linear approximation [15] were proposed. These approaches are effective only for some special cases that image intensity distribution were simple 
and fail for complicated cases. Because of non-uniform image density distribution in blood vessel ultrasound images, the usage of MS model fails to segment blood vessels properly.

To overcome this failure in this research we used from MS model introduced by Xiaojun and Tien [7] which is a modified version of original MS model. In new model, the energy function is given by

$$
E(C)=\int_{\Omega \backslash C}\left|F\left(u_{0}\right)\right|^{2} d x d y+\mu|C|
$$

where $F\left(u_{0}\right)$ is the high frequency components of the original image and $\mu$ is balance parameter. The new minimal energy function consists of high frequency components of regions and the length of segmentation curve. If we minimize the energy function, only low frequency components will be exists inside regions and high frequency parts are preserved in boundaries. The noise effect is suppressed by curve length constraint.

In new energy function, only we have a one variable that should be solved and it is segmentation curve length. This model provides two main advantages for us to conduct blood vessel segmentation problem. First, only one equation should be solved and then this is a fast algorithm in image segmentation. This advantage lets us to extract the vessel movement profile in an ultrasound movie in addition to extract vessel boundaries. Second, because no constant and linear approximations are used, the model can deal in problems with different image density distributions such as blood vessels ultrasound images. To improve blood vessel detection, a wavelet based filter using Gabor transform is applied. In next section, we will describe the Gabor wavelet filter.

\section{GABOR WAVELET}

A Gabor function is a complex sinusoid which is modulated by a rotated Gaussian function. Bovik et al [16] suggest the restriction of the choice of Gabor filters to those with isometric Gaussians (aspect ratio of one). The spatialdomain presentation off the corresponding Gabor function is

$$
g_{u, v}(x, y)=\frac{\left\|k_{u, v}\right\|^{2}}{2 \pi \sigma^{2}} e^{-\frac{\left\|K_{u, v}\right\|^{2}\left(x^{2}+y^{2}\right)}{2 \sigma^{2}}}\left[e^{i k_{u, v}(x+y)}-e^{-\frac{\sigma^{2}}{2}}\right]
$$
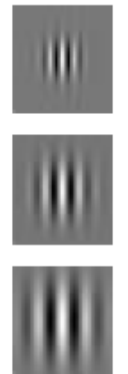

$\mu=0$,
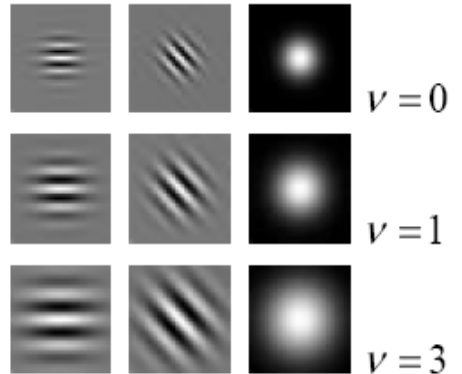

2 ,

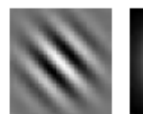

3
Fig. 1. Gabor wavelets for $u \in\{0,1,2,3\}$ and $v \in\{0,1,3\}$

where $k_{u, v}$ is wave vector defined by both orientation $u$ and scale $v$ and defined as $k_{u, v}=k_{v} e^{i \psi_{u}}$. In equation 3 , the first term in square brackets is the oscillatory part of Gabor function. The parameter $\sigma$ defines the ratio of Gaussian window width and wavelength. Also we have

$$
\begin{gathered}
k_{v}=\frac{\pi}{2(\sqrt{2})^{v}} \\
\psi_{u}=\frac{\pi u}{4}
\end{gathered}
$$

Fig. 1 shows the real part of Gabor kernels with $\sigma=2 \pi$ for $v=[0,1,3]$ and $u=[0,1,2,3]$. In this research, to accelerate the speed of algorithm, only imaginary parts of Gabor wavelet used. Also the best value of $\sigma$ is determined as $\sigma=\pi$. Also in order to accelerate the algorithm speed, only one orientation $(u=3)$ and one scale $(v=1)$ is used. Using these parameters, results are still good enough to be used as second stage on blood vessel segmentation process.

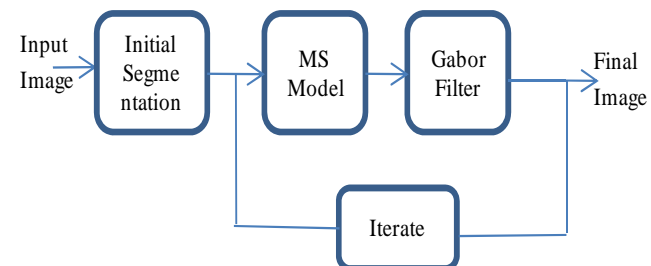

Fig. 2. Overall structure of blood vessel segmentation algorithm

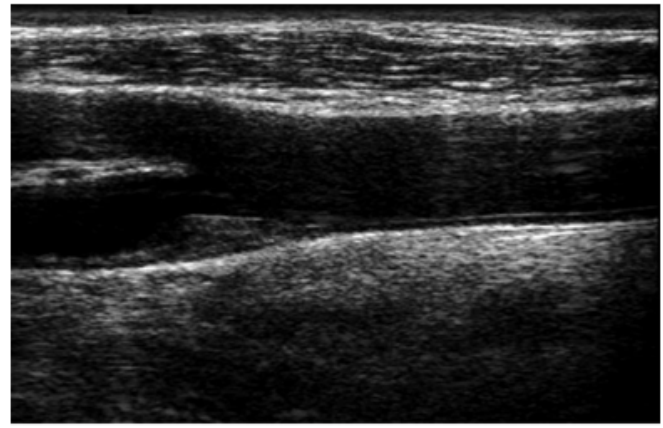

(a)

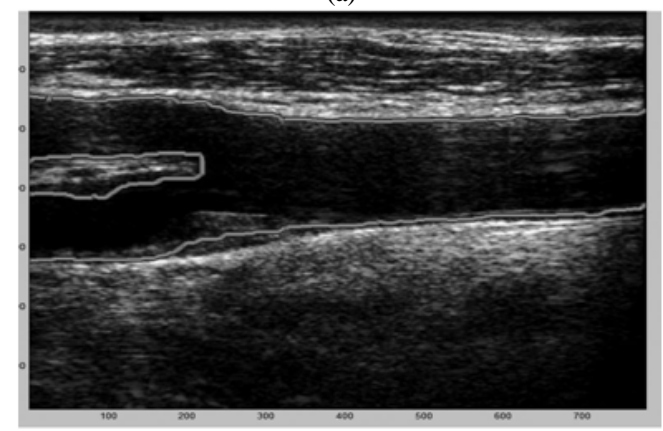

(b)

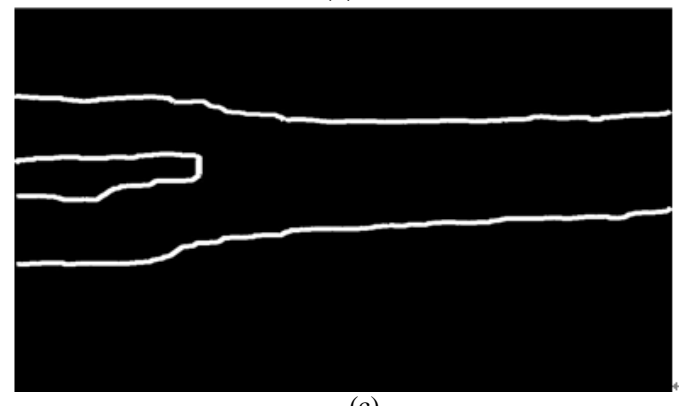

(c)

Fig. 3. (a) Original ultrasound image, (b) final Image segmentation image, (c) final detected boundary.

\section{Two Stage Image Segmentation Algorithm}

In this section, proposed two stage image segmentation 
algorithm will be described. Fig. 2 shows the overall structure of introduced method for segmentation of blood vessels. As shown in figure2, because of non-uniform illumination in blood vessels, an adaptive threshold is used to create an initial segmentation. As, the results of initial segmentation are not good and there is noise in different regions, the created initial segmentation is fed into an iterative process. This process includes two stages. In first step, segmentation is done by applying MS model and the output of first step is corrected by a Gabor filter.

\section{EXPERIMENTAL RESULTS}

\section{A. Ultrasound Image Database}

We couldn't find a standard database for heart vesselsultrasound images. In this research we extracted our images from 10 brachial vessels ultrasound movies from Tehran Heart Center. Using these ultrasound movies, finally a heart vessels image database with 120 images was created.

\section{B. Experiments}

In the process of image segmentation, as described in section III, only imaginary part of Gabor filter is used in image segmentation. To accelerate the computation, in each iteration, only narrow band Gabor filter is calculated beside the segmentation curve from MS model. As a result, the segmentation process is relatively fast. Training step is the most time consuming step in image segmentation problems. As our method needs no training step, the segmentation process is fast enough to be used in processing of ultrasound image series.

We tested our approach on 120 images of our database. Fig. 3 shows the original ultrasound image, final boundary detection and final segmentation of blood vessels from ultrasound image.

\section{CONCLUSION}

In this research, we use a new image segmentation model to segment and detect boundaries of heart vessels images. The proposed method is based on a modified version of Mumford-Shah model. MS model is a region based approach that uses from segmentation curves. The MS model is a good segmentation approach, but the minimization of original energy function in not a trivial task and needs an approximation stage. However, due to nonuniform image density distribution in complicated case, approximation techniques such as constant and linear methods can not deal with such cases. In used modified MS model, this problem is solved by applying new energy function that could be easily solved with one PDE and can handle with complicated cases such as blood vessels images.

In order to improve segmentation results, a Gabor filter is applied after MS model. To accelerate the speed of algorithm, we used only a simplified version of Gabor filter, but results are still good and algorithm is fast relatively in comparison with other segmentation approaches. We are working now to generalize this approach that could be used in ultrasound movies to extract the vessel movement profile.

\section{REFERENCES}

[1] P. Agostoni, J. A. Schaar, and P. W. Serruys, "The Challenge of Vulnerable Plaque Detection in the Cardiac Catheterization Laboratory,” ardiovaskuläre Medizin, pp. 349-358, Jul. 2004.

[2] Y. A. Tolias and S. M. Panas, "A fuzzy vessel tracking algorithm for retinal images based on fuzzy clustering," IEEE Trans. Med. Imag, vol. 17, no. 2, pp. 263-273, Apr. 1998.

[3] T. McInerney and D. Terzopoulos, "T-snakes: Topology adaptive snakes,” Med. Image Anal., vol. 4, pp. 73-91, 2000.

[4] J. J. Staal, M. D. Abràmoff, M. Niemeijer, M. A. Viergever, and B. V. Ginneken, "Ridge based vessel segmentation in color images of the retina,” IEEE Trans. Med. Imag., vol. 23, no. 4, pp. 501-509, Apr. 2004.

[5] J. V. B. Soares, J, J, G. Leandro, R. M. Cesar, H. F. Jelinek, and M. J. Cree, "Retinal Vessel Segmentation Using the 2-D Gabor Wavelet and Supervised Classification,” IEEE Trans. Med. Imag, vol. 25, no. 9, pp. 1214-1222, Sep. 2006.

[6] D. Mumford and J. Shah, "Optimal approximation by piecewise smooth functionals and associated variational problems," Comm. Pure Appl. Math, vol. 42, pp. 577-685, 1989.

[7] X. Du and T. D. Bui, "Retinal Image Segmentation Based on Mumford-Shah Model and Gabor Wavelet Filter," in Proc. of 2010 20th International Conference on Pattern Recognition ICPR, pp. 3384-3387, , 2010

[8] H. Wei and M. Bartlets, "Unsupervised Segmentation Using Gabor Wavelets and Statistical Features in LIDAR Data Analysis," in Proc. of 18th International Conferences On Pettern Recognition, September 2006, pp. 667-670.

[9] L. Ambrosio, "Existence theory for a new class of variational problems,” Arch. Rat.Mech. Anal, vol. 111, pp. 291-322, 1990.

[10] G. Congedo and I. Tamanini, "On the existence of solutions to a problem in multidimensional segmentation,” Ann. Inst. H. Poincar'e, Anal. Nonlin. 8, pp. 175-195, 1991

[11] G. D. Maso, J. M. Morel, and S. Solimini, "A variational method in image segmentation:Existence and approximation results,” Acta Math, no. 168 , pp. 89-151, 1992.

[12] S. Gao and T. D. Bui, "Image segmentation and selective smoothing by using Mumford-Shah model," IEEE Trans on Image Processing, vol. 14, no. 10, pp. 1537-1549, 2005.

[13] L. Vese and T. F. Chan, "A multiphase level set framework for image segmentation using the Mumford and Shah model," International Journal of Computer Vision, vol. 50, no. 3, pp. 271-293, 2002.

[14] T. F. Chan and L. A. Vese, "Active contours without edges," IEEE Trans on Image Processing, vol. 10, no. 2, pp. 266-277, 2001.

[15] T. D. Bui, S. Gao, and Q. H. Zhang, "A generalized Mumford-Shah model for roof-edge detection," IEEE International Conference on Image Processing, vol. 2, pp. 1214-1217, 2005.

[16] A. C. Bovik, M. Clark, and W. S. Geisler. "Multichannel Texture Analysis Using Localized Spatial Filters," IEEE Transactions on Pattern Analysis and Machine Intelligence, vol. 12, no. 1, pp. 55-73, 1990 